\title{
Lernen von Marx und Lenin?
}

\author{
Michael Aßländer
}

Der Diagnose des Status Quo der Wirtschaftsethik in West- und Osteuropa von Franz Rupert Hrubi ist in weiten Teilen zuzustimmen: Das Interesse an einer Wirtschaftsethik in Westeuropa ist im Stagnieren begriffen, die realen Probleme des Transformationsprozesses in Osteuropa lassen die Frage nach der Wirtschaftsmoral in den Hintergrund treten und die Suche nach moralischen Leitbildern, an die eine Wirtschaftsmoral in Osteuropa anknüpfen könnte, bleibt, wie es scheint, weithin ergebnislos. Ebenso zuzustimmen ist Franz Rupert Hrubi auch hinsichtlich des Beharrungsvermögens kommunistischer Denktraditionen im Bewußtsein der neuen politischen und ökonomischen Entscheidungsträger. Kurz und gut: Seine Zustandsbeschreibung verheißt wenig Anlaß zur Freude.

Dabei verweist aber gerade die Schwierigkeit, sich innerhalb des Transformationsprozesses, dem sich viele Osteuropäer wie einer über sie hereinbrechenden Naturgewalt hilflos ausgeliefert sehen, zurecht zu finden, auf das Fehlen ethischer und moralischer Orientierungspunkte. Das Lamento, daß die vierzig- bzw. siebzigjährige Herrschaft des real existierenden Sozialismus zur Erosion demokratischer und sozialer Standards - so denn überhaupt vorhanden - geführt haben, nützt an dieser Stelle wenig. Auch der allenthalben übliche Verweis auf die Selbstheilungskräfte freier Märkte erfüllt den unvoreingenommenen Betrachter angesichts der drohenden sozialen Destabilisierung insbesondere der GUS-Staaten eher mit Skepsis als mit Zuversicht.

Angesichts dieser Ausgangslage scheint es wenig sinnvoll, eine Wirtschafts- und Unternehmensethik etablieren $\mathrm{zu}$ wollen, die in ihren Grundlagen an Werthaltungen gebunden ist, für die dem ehemaligen Bürgern des Ostblocks jegliches Verständnis fehlen muß. Weder kann hier an eine Tradition privaten Unternehmertums angeknüpft werden, noch auf eine liberale Wirtschaftsgesinnung mit ihren Werten wie Freizügigkeit, Subsidiarität oder Eigenverantwortlichkeit Bezug genommen werden. Wenn es also darum geht, „das Eisen zu schmieden, solange es heiß ist" - wie Professor Hrubi sich ausdrückt -, dann gilt es die Frage nach dem möglichen Ausgangspunkt für eine Wirtschaftsethik gerade in Osteuropa neu zu überdenken.

Drei Punkte scheinen mir an dieser Stelle besonders bemerkenswert:(1) Es hat den Anschein, daß der Begriff „Transformation“ impliziert, es handele sich beim Übergang zu einer neuen Wirtschafts- und Gesellschaftsstruktur lediglich um eine kurze Übergangsphase, die es in Kauf zu nehmen gilt, auf diesem Wege zu 
einer neuen (und besseren) Gesellschaft. Somit konzentrieren sich die meisten Ratschläge in Bezug auf Wirtschaftsethik und Wirtschaftspolitik allenthalben darauf, Weichenstellungen für die zukünftige Gesellschaft vorzunehmen. Außer acht bleiben dabei Empfehlungen für den Transformationsprozeß selbst. Wie Lobomír Mlcoch, Dekan an der Sozialwissenschaftlichen Fakultät der Karlsuniversität in Prag, betont, glauben viele ehemalige Ostblockstaaten einem falsch verstandenem Liberalismus folgen und erfolgreiche Privatisierung mit erfolgreichem Übergang zur Marktwirtschaft gleichsetzen zu müssen. Ziel des Transformationsprozesses scheint es daher zu sein, nach der Zerschlagung der bisherigen Wirtschaftsstrukturen eine exakte Kopie westlicher Wirtschaftsordnungen zu implementieren. ${ }^{1)}$ Dabei wird jedoch übersehen, daß die Konsolidierungsphase der sozialen Werthaltungen und die Stabilisierung gesellschaftlicher Normen einen wesentlich längeren Zeitraum in Anspruch nehmen, als es die vermeintlich schnelle Umstellung auf private Wirtschaftsstrukturen vermuten läßt. Insgesamt scheint der „kurze Weg“ einer radikalen Umstellung auf private Wirtschaftsstrukturen nicht weniger Zeit in Anspruch zu nehmen als der „lange Weg“ eines allmählichen Übergangs. ${ }^{2)}$

(2) Die zeitgleiche Aufhebung der staatlichen und der wirtschaftlichen Ordnung führt zum völligen Verlust der Steuerbarkeit des Transformationsprozesses. Gerade das Fehlen ordnungspolitischer Instanzen, ausgestattet mit den entsprechenden Machtbefugnissen, bedingt jenes mangelnde Vertrauen in die neuen "Wirtschaftsstrukturen“, das Franz Rupert Hrubi in seinem Artikel beklagt. Ohne entsprechende staatliche Ordnungsinstanzen als Garanten einer vorhersagbaren stetigen Wirtschaftspolitik lassen sich seitens der Wirtschaft keine stabilen Zukunftserwartungen aufbauen. Die ungenügende Sicherheit der Investoren, die mangelnde Vorhersagbarkeit zukünftiger Entwicklungen und die fehlende Rechtssicherheit münden so in einen „Casino-Kapitalismus“, dessen einziges Ziel die Realisation kurzfristiger Gewinne, nicht aber der Aufbau dauerhafter Wirtschaftsstrukturen ist. Was bleibt ist eine „hit-and-run-Strategie“, die die einmalige Gewinnmitnahme zum Ziel hat, ohne an langfristigen Geschäftsbeziehungen interessiert $\mathrm{zu}$ sein. In Folge davon kommt es zwangsläufig zur Erosion moralischer Standards, da ausschließlich Gewinn und nicht langfristige Partnerschaft zum Ziel der Geschäftsbeziehungen werden. Zudem ergeben sich insbesondere aus der mangelnden Rechtssicherheit bedenkliche Nebeneffekte: Der mangelnde Schutz der Eigentumsrechte und die ungenügende Garantie langfristiger Renditen führt $\mathrm{zu}$ individuellen

\footnotetext{
1 ) Vgl. Mlcoch, L.: A Criticism of Misunderstood Liberalism, in Journal of Business Ethics 9-10/1998, S. $953 \mathrm{f}$.

2 ) Vgl. ebd., S. 953.
} 
Mechanismen zum Schutz des Privateigentums, die das Eindringen von Gewalt erleichtern und mafiöse Strukturen in der Wirtschaft begünstigen. ${ }^{3)}$

(3) Das Fehlen einer staatlichen Wirtschafts- und Wettbewerbskontrolle hat weitreichende Folgen auch im Bereich der Unternehmensgründungen. So benachteiligt der mangelnde staatliche Schutz Kleinanleger gegenüber den Hauptinvestoren größerer Aktiengesellschaften. Kleinaktionäre können sich vielfach gegen die Diskriminierung durch kapitalkräftigere Mitgesellschafter nicht effektiv zur Wehr setzen. Wie bereits Walter Eucken betonte, führt hier die Garantie von Freiheitsrechten innerhalb der Wirtschaft durch die Möglichkeit der Machtagglomeration zur faktischen Aufhebung dieser Freiheitsrechte. Durch Kapitalkonzentration sind einige wenige in der Lage, andere zu dominieren und in ihren Freiheiten einzuschränken. Eucken fordert daher eine aktive staatliche Wettbewerbspolitik, die diese Form der Machtkonzentration dauerhaft zu verhindern in der Lage ist. ${ }^{4)}$ Diese mangelnde Kontrolle wirtschaftlicher Machtkonzentrationen wirkt sich in Zeiten der Transformation insbesondere deshalb negativ auf die wirtschaftliche Umstrukturierung aus, da sie neben dem fortlaufenden Vertrauensverlust des Mittelstandes in die Wirtschaft auch gerade jene Denkstrukturen unterstützt, wie sie für die Strategienbildung in planwirtschaftlich organisierten Großbetrieben maßgeblich waren. Das von Professor Hrubi konstatierte Beharrungsvermögen planwirtschaftlicher Denkstrukturen, hat nicht zuletzt hierin zugleich seinen Ausdruck und seine Ursache.

Es bleibt somit die Frage, welche wirtschaftsethischen Empfehlungen sich an eine Ökonomie im Stadium des Transformatinsprozesses geben lassen. Das hauptsächliche Charakteristikum derartiger Gesellschaften scheint mir zu sein, daß zwar einerseits die wirtschaftlichen, sozialen und politische Strukturen einem fundamentalen Wandel unterzogen wurden, andererseits aber noch kein adäquates soziales und politisches Bewußtsein existiert, das es ermöglichen könnte, jene Werthaltungen zum Ausdruck zu bringen, die für das Funktionieren dieser neuen Ordnung maßgeblich sind. Wie also lassen sich neue Strukturen und alte Denkmuster miteinander in Einklang bringen?

Ein Versuch, dieses Problem zu lösen, dem ich mich, wenn auch nicht in allen Punkten, anschließen möchte, stammt von Angelo Corlett von der San Diego State University. Seine Kernfrage lautet nicht: „Was muß sich ändern, damit der Weg zu neuen Denkmustern frei wird?“, sondern „Welchen Beitrag kann eine Marxistische Philosophie zur Wirtschaftsethik leisten?“. Corlett kommt dabei zu

3 ) Vgl. ebd., S. 955.

${ }^{4}$ ) Vgl. Eucken, W.: Die Wettbewerbsordnung und ihre Verwirklichung, in Eucken, W. und Böhm, F. [Hrsg.]: Ordo - Jahrbuch für die Ordnung von Wirtschaft und Gesellschaft. Würzburg 1949, S. 64 - 83. 
folgendem Ergebnis: (1) Auf einer deskriptiven Ebene bildet die mangelnde Fähigkeit des Wirtschaftsliberalismus, gesellschaftliche Werthaltungen, moralische Verantwortung und Persönlichkeitsrechte zum Ausdruck zu bringen, den zentraler Kritikpunkt einer „marxistischen Wirtschaftsethik“. Neu zu etablierende Wirtschaftsstrukturen sind in diesem Sinne auf ihre "Gesellschaftsverträglichkeit" hin $\mathrm{zu}$ analysieren und gegebenenfalls $\mathrm{zu}$ modifizieren. (2) Auf einer normativen Ebene geht es einer marxistischen Philosophie, im Gegensatz zum klassische Wirtschaftsliberalismus, vor allem um die gesellschaftliche Perspektive. Dabei stehen die Verantwortung des einzelnen gegenüber der Gesellschaft und für die Gesellschaft ebenso im Zentrum der Betrachtungen wie Fairneß und soziale Gerechtigkeit. Sozioökonomische und politische Strukturen müssen nicht nur nach Maßgabe ihres wirtschaftlichen Erfolges sondern auch nach Maßgabe ihrer Fähigkeiten zu gerechtem Interessenausgleich gemessen werden. (3) Anliegen einer marxistischen Wirtschaftsethik nach Corlett ist schließlich ebenso die gerechte Verteilung gesellschaftlicher Mitspracherechte. Um die einseitige Machtagglomeration auf Seiten des Kapitals zu verhindern, fordert er eine paritätische Besetzung unternehmerischer Entscheidungsgremien durch Kapitaleigner und Arbeitnehmer. ${ }^{5)}$

Allgemein gesehen scheint eine derartige Philosophie durchaus in der Lage, den oben angeführten Defiziten von „Transformationsgesellschaften“ entgegenzuwirken. Betrachtet man also die marxistische Philosophie nicht als starre Doktrin, sondern als lernfähige Gesellschaftstheorie, die durchaus in der Lage ist, auf geänderte Rahmenbedingungen zu reagieren, ergibt sich hier durchaus die Möglichkeit, nicht nur einen ökonomischen, sondern durchaus auch einen gesellschaftstheoretischen Transformationsprozeß einzuleiten. Jedenfalls scheint eine kritische marxistische Philosophie durchaus in der Lage, sich innerhalb des Transformationsprozesses als Ausgangspunkt für gesellschaftliche Veränderungen zu bewähren.

5 ) Vgl. Corlett, A. J.:A Marxist Approach to Business Ethics, in Journal of Business Ethics 1/1998; S. $100 \mathrm{f}$. 